\title{
Study on Rigid-Flexible Coupling Dynamic Model of Globoid Cam Indexing Mechanism with Six Degrees of Freedom
}

\author{
Zhenghao $\mathrm{Ge}^{1, \text { a }}$, Chen Liang ${ }^{1, \mathrm{~b}}$ Chengping $\mathrm{Li}^{1}$ \\ ${ }^{1}$ Shaanxi University of Science and Technology, Xi'an Shaanxi, 710021,China. \\ agezh@sust.edu.cn, bliangchen1027@163.com
}

\begin{abstract}
Keywords: Globoid indexing cam mechanism, Rigid-flexible coupling, Dynamic Analysis Dynamic
\end{abstract} model

\begin{abstract}
Aiming at cam indexing mechanism, this paper, considering the influence of elastic deformation, bending and twisting factors, combining the knowledge of rigid-flexible coupling Dynamic System, established the rigid-flexible coupling dynamic model of globoid cam indexing mechanism with six degrees of freedom. Then listed the equation of dynamic characteristic equation and got the equation of natural frequency. Finally establish the 3D model in software. Which offers remarks and suggestions for the dynamic characteristic analysis of cam mechanisms.
\end{abstract}

\section{Introduction}

All manuscripts must be in English. Please keep a second copy of your manuscript in your office (just in case anything gets lost in the mail). When receiving the manuscript, we assume that the corresponding authors grant us the copyrig While cam is achieving work cycle of dynamic/static alternation, the motion law of follower will generate distortion by excitation that whole cam indexing mechanism received, and because the inertial load, caused by periodic intermittent motion, gives the mechanism periodic vibration, some difference will come into being between theoretical values and mechanism output dynamic response. Just improving geometric accuracy cannot ensure that the system has favorable dynamic characteristics; in order to eliminate residual vibration in a very short cycle period and achieve precise location, this dynamic problem has become a major research subject at home and abroad. In order to research actual dynamic model of globoid cam indexing mechanism, this paper trys to establish a kind of equivalent model to effectively simulate real situation of globoid cam indexing mechanism; combining with relevant knowledge of Mechanical Vibration Course and Elasticity, this paper establishes the rigid-flexible coupling dynamic analysis equation of globoid cam indexing mechanism with six degrees of freedom.

\section{The establishment of the dynamic model}

\subsection{The rigid-flexible coupling dynamic model}

In traditional mechanical system dynamic analysis, we assumed that all the members are rigid body, namely Mechanical Rigid-body Dynamic System. But with the rising speed of mechanical movement, losing weight of machine itself and the improving of working accuracy, the elasticity of members always becomes a non-negligible factor. We call mechanical system composed by elastomers as mechanical flexible dynamic system. Normally if elastic system is dispersed into discrete systems with finite degree of freedom, the system's degree of freedom will also be greatly increased, which improves the model precision of system. Due to the existence of elastic deformation, the potential energy of system must be taken into consideration; and the excessive degree of freedom will bring more parameters, which increases the difficulty in solving the equation.

Comprehensively considered the advantage and disadvantage of Mechanical Rigid-body Dynamic System and Mechanical Flexible Dynamic System, people creatively developed rigid-flexible coupling Dynamic System. Assuming members which are prone to torsional deformation and bending deformation in high speed working condition or members which require very high precision 
as elastic body, and assuming members which are not easy to get deformation or members which require not very high precision as rigid body, in this way, we can not only simulate actual dynamic performance of mechanism, but also decrease the difficulty in establishing and solving the equation of dynamic model. At present, the rigid-flexible coupling dynamic model is gradually attracting more attention.

\subsection{The foundation for the establishment of dynamic model,}

As for globoid cam indexing mechanism, we use the lumped mass method, and establish multi-degree of freedom dynamic model. The method of lumped mass means that the real mechanical system is discretized as discrete system which has several degrees of freedom and several mass points, and each point has continuous distributed mass $(\mathrm{m})$ 、 stiffness $(\mathrm{k})$ 、 damp (c) and so on. Considering the torsional vibration mode of cam, the torsional vibration mode and translational vibration mode of indexing plate and loading plate, we establish globoid cam indexing mechanism multi-degree of freedom dynamic model, such as Figure 1.

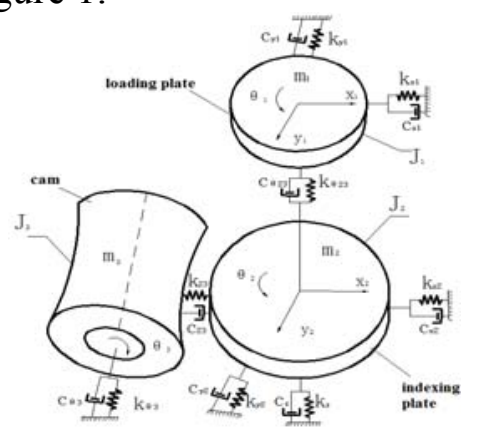

Fig.1, globoid cam indexing mechanism multi-degree of freedom dynamic model

The stiffness of cam, indexing plate and rollers in globoid cam indexing mechanism is very high, so the deformation usually can be ignored.

\subsection{The establishment of the rigid-flexible coupling dynamic model}

The globoid cam indexing mechanism is composed of input shaft (driving shaft), output shaft (driven shaft), globoid cam, indexing plate, rollers, and working position plate. Among them, the input shaft and output shaft, under the effect of various strengths, will generate elastic deformation, namely torsional deformation and bending deformation. The torsional deformation will immediately affect positioning accuracy of rotary plate. Although the bending deformation will affect the meshing between cam and roller, the deflection of meshing part is very small, which can be ignored. The input shaft usually is shorter, and with higher stiffness, so we can also ignore the deformation. Therefore, the globoid cam indexing mechanism can be abstracted to a rigid-flexible coupling torsional vibration system in which input shaft and output shaft are flexible bodies while other members are rigid bodies. Therefore, we can consider the part of loading plate and output shaft as a mass body to do dynamic research using the method of lumped mass.

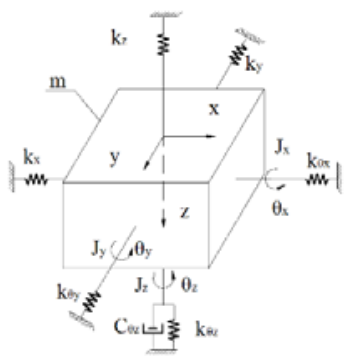

Fig.2, the equivalent mass body dynamic model of cam mechanism

In Figure 2, the mass body which composed by loading plate and output shaft has six degrees of freedom, including the vibration in $\mathrm{x} / \mathrm{y} / \mathrm{z}$ axes, and the rotation around $\mathrm{x} / \mathrm{y} / \mathrm{z}$ axes, and among them, the rotational inertia of the mass rotating around the axis $\mathrm{X}$ is ${ }^{J_{x}}$, the rotational inertia of the mass rotating around the axis $\mathrm{Y}$ is ${ }^{J_{y}}$, and the rotational inertia of the mass rotating around the axis $\mathrm{Z}$ is $J_{z}$. The loading plate and output shaft are revolution body which rotate around the axis $Z$, so $J_{x} \approx J_{y}$ 
About the equivalent mass body dynamic model, according to Lagrangian Method, we can establish the dynamic equations of globoid cam indexing mechanism. Due to nonlinear factors produced by torsional deformation, we should do linear processing.

$\tau\left(\theta_{x}\right) \approx \tau(\theta)+\tau^{\prime}\left(\theta_{x}-\theta\right)$

Where: $\tau^{\prime}=\frac{d \tau}{d \theta}$

(1) The vibration equation about equivalent mass body in direction of $\mathrm{x}$.

$m \ddot{x}+k_{x} x=0$

(2) The vibration equation about equivalent mass body in direction of $y$.

$m \ddot{y}+k_{y} y=0$

(3) The vibration equation about equivalent mass body in direction of $z$.

$m \ddot{z}+k_{z} z=0$

(4) The torsional vibration equation about equivalent mass body around the axis $\mathrm{x}$.

$m \phi_{x}+k_{\theta x} q_{x}=J_{x}(\theta)$

(5) The torsional vibration equation about equivalent mass body around the axis y.

$m \ddot{q}_{y}+k_{\theta y} q_{y}=J_{y} \ddot{\tau}(\theta)$

(6) The torsional vibration equation about equivalent mass body around the axis z.

$m \ddot{q}_{z}+C_{\theta z} \dot{q}_{z}+k_{\theta z} q_{z}=J_{z} \ddot{\tau}(\theta)$

Where:

$\tau 、 \approx 、$ were equivalent mass body theory rotation, angular velocity and angular acceleration respectively;

$\theta_{x} 、 \theta_{y} 、 \theta_{z}$ were equivalent mass body real rotation, angular velocity and angular acceleration respectively;

$q_{x} 、 q_{y} 、 q_{z}$ were equivalent mass body rotation around axis $\mathrm{x}, \mathrm{y}$ and $\mathrm{z}$ respectively, as they were small, they can be equivalent to linear displacement.

$q_{x}=\theta_{x}-\tau(\theta)$;

$q_{y}=\theta_{y}-\tau(\theta)$;

$q_{z}=\theta_{z}-\tau(\theta)$

$x, y, z$ were equivalent mass body linear displacement in direction of $\mathrm{x}, \mathrm{y}, \mathrm{z}$ respectively.

$k_{x} 、 k_{y} 、 k_{z}$ were equivalent mass body equivalent bending rigidity;

$k_{\theta x} 、 k_{\theta y} 、 k_{\theta z}$ were equivalent mass body equivalent torsional stiffness around axis $\mathrm{x}, \mathrm{y}, \mathrm{z}$ respectively.

$C_{\theta z}$ was equivalent mass body damping coefficient around axis z.

The loading plate and output shaft are axisymmetric body around axis $\mathrm{z}$, so the bending rigidity is similar to torsional stiffness in direction of $\mathrm{x}$, y. so $k_{x} \approx k_{y}, k_{\theta x} \approx k_{\theta y}$

\subsection{The parameters of dynamic mathematical model equation}

According to the theory of mechanical system dynamics, mechanism system dynamics equation:

$M \ddot{X}+C \dot{X}+K X=P$

Substitute the expression (2) to (7) into expression (8), and then we can get the parameter matrix about the globoid cam indexing mechanism dynamic equation, as follows:

Generalized coordinates matrix 


$$
X=\left[\begin{array}{llllll}
q_{x} & q_{y} & q_{z} & x & y & z
\end{array}\right]^{T}
$$

According to graph 1, while cam is rotating clockwise, the indexing plate was driven to rotate anticlockwise. The linear displacement, which is along the positive direction of coordinate axis in generalized coordinates matrix, is positive, and the anticlockwise is the angle positive of output shaft system.

(2) Mass matrix

$M=\left[\begin{array}{llllll}m & m & m & m & m & m\end{array}\right]$

(3)Damping matrix

Various damping will have impact on the cam mechanism while it is working, normally the damping can be ignored, but when exciting frequency is close to natural frequency, the influence of damping will be more significant; in this situation, we should take the influence of damping into consideration, and vibration mode is as follows:

$C=\left[\begin{array}{llllll}C_{1} & C_{2} & C_{3} & C_{4} & C_{5} & C_{6}\end{array}\right]$

According to vibration mode:

$\Phi^{T} C \Phi=\bar{C}=\left[\begin{array}{cccc}2 \zeta_{1} \omega_{1} & & & 0 \\ & 2 \zeta_{2} \omega_{2} & & \\ & & 0 & \\ 0 & & & 2 \zeta_{n} \omega_{n}\end{array}\right]$

Where $\Phi$ is positive definite matrix, $\bar{C}$ is diagonal matrix. $\zeta_{i}(i=1,2 \cdots n)$ is vibration mode damping ratio in $i$ step. So the damping matrix of the globoid cam indexing mechanism:

$C=\left[\begin{array}{llllll}0 & 0 & C_{\theta z} & 0 & 0 & 0\end{array}\right]$

(4)Inertia load matrix

The mainly load in mechanism system are inertia force and friction force, as the friction force is so low that it can be ignored, so we just consider inertia force; therefore, the inertia load matrix of globoid cam indexing mechanism is:

$P=\left[\begin{array}{llllll}-J_{x} & -J_{y}(\theta) & -J_{z} & 0 & 0 & 0\end{array}\right]$

(5)Stiffness matrix

$$
K=\left[\begin{array}{cccccc}
k_{\theta x} & 0 & 0 & 0 & 0 & 0 \\
0 & k_{\theta y} & 0 & 0 & 0 & 0 \\
0 & 0 & k_{\theta z} & 0 & 0 & 0 \\
0 & 0 & 0 & k_{x} & 0 & 0 \\
0 & 0 & 0 & 0 & k_{y} & 0 \\
0 & 0 & 0 & 0 & 0 & k_{z}
\end{array}\right]
$$

\subsection{Calculation of natural frequency about dynamic model}

According to above-mentioned dynamic parameters about the globoid cam indexing mechanism, using modal superposition method to solve the matrix equation about expression (8), we can get the relative dynamic data.

When using modal superposition method to solve problems, we discrete time to unit time. In each unit, the cam mechanism can be regarded as time-invariant system, thus:

$$
\left[K-\omega^{2} M\right] \phi=0
$$

Solving expression (16) we can get natural frequency and vibration mode vectors at each orders in this unit time, $n$ is vibration mode number, in this case $n=6$.

Substitute the expression (15) into expression (16), and then we can get the natural frequency equation of globoid cam indexing mechanism: 


$$
\left[\begin{array}{cccccc}
k_{\theta x}-\omega^{2} m & 0 & 0 & 0 & 0 & 0 \\
0 & k_{\theta y}-\omega^{2} m & 0 & 0 & 0 & 0 \\
0 & 0 & k_{\theta}-\omega^{2} m & 0 & 0 & 0 \\
0 & 0 & 0 & k_{x}-\omega^{2} m & 0 & 0 \\
0 & 0 & 0 & 0 & k_{y}-\omega^{2} m & 0 \\
0 & 0 & 0 & 0 & 0 & k_{z}-\omega^{2} m
\end{array}\right]=0
$$

\section{The establishment of the rigid-flexible coupling model}

\subsection{The establishment of the multi rigid body model}

In globoid cam indexing mechanism, 3D modeling is a difficult point, parametric modeling is a more accurate and simple way.

Firstly, based on profile equation, we use programming language to calculate the 3D coordinate of several point on sweeping curves, and save it as ".ibl" file, then we introduce it into 3D software to create corresponding curves, finally we get the cam model, such as Figure 3.

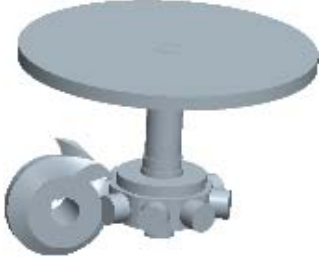

Fig.3, the 3D modeling of globoid cam indexing mechanism

We build the 3D modeling of cam mechanism in Pro/E, and use MCHE/PRO to introduce it into ADAMS, and then the model just includes geometric features and position relationship of several parts. So we need to define the parameters of model in Adams/View. The globoid cam indexing mechanism dynamic model should include: gravity field, mass, constraint, driving.

By setting all kinds of parameters, we finally form the virtual prototype such as Figure 4:

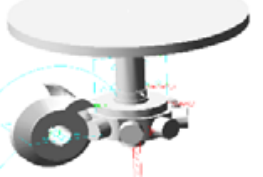

Fig.4, the multi rigid body virtual prototype of globoid cam indexing mechanism

\subsection{The establishment of the rigid-flexible coupling model}

In globoid cam indexing mechanism, the input shaft usually is shorter, and with higher stiffness, so we can also ignore the deformation. Therefore, the globoid cam indexing mechanism can be abstracted to a rigid-flexible coupling torsional vibration system in which input shaft and output shaft are flexible bodies while other members are rigid bodies. We use the ANSYS to establish the flexible body. The basic steps are as follows:

(1)Build the 3D model. Establish the member model, derived as “.X_T" file.

(2)Introduce the ".X_T" file into ANSYS, make model analysis, solving the natural frequency and vibration mode of this member.

Derive this file as ".mnf” file.

(3)Open the globoid cam indexing mechanism multi rigid body model in ADAMS, using the rigid to flex tool to introduce the ".mnf" into ADAMS to replace the rigid body model. Check the model, confirm that the mark points of flexible shaft and rigid shaft completely coincide. Add the constraint, kinematic pair, driving and other boundary conditions in rigid-flexible coupling model. By this way, we can establish the complete rigid-flexible coupling dynamic virtual prototype, such as Figure 5: 


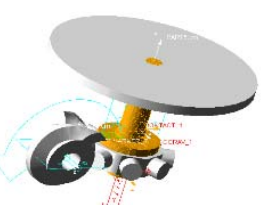

Fig.5, the rigid-flexible coupling dynamic virtual prototype

\section{Conclusion}

This paper focuses on cam indexing mechanism and starts from mechanical vibration theory and mechanical system dynamic analysis. Taking bend and torsional into consideration, this paper combines the rigid-flexible coupling theory, establishes the rigid-flexible coupling dynamic model of globoid cam indexing mechanism with six degrees of freedom, and gets the equation of solving natural frequency, which offers theoretical basis for the prediction and estimation of dynamic characteristics.

\section{Reference}

[1]Chang Zongyu, Zhang Ce, Yang Yuhu and Wang Yuxin. Dynamic Modeling on Globoid Indexing Cam Mechanism Using Kane Equation [J].Chinese Journal of Mechanical Engineering, 2001, 03:34-37+40.

[2]Li Lei, Feng Xianying, Zhang Chengliang, Mou Shigang, Wang Suiyuan. Dynamic Model and Modal Analysis of Ball Bearing Globoidal Indexing Cam Mechanism [J]. Journal of Vibration and Shock, 2012, 16:62-65+76.

[3]Miao Miao, Tao Xueheng, Jing Jing, Feng Shaoling. Dynamic Model of Globoidal Indexing Cam Mechanism of 5-Degrees of Freedom with Double-Hemisphere Rolling Teeth. [A]. Professional Committee of Mechanism, Mechanical Transmission Institution of Chinese Mechanical Engineering Society. Symposium on the National Seminar of Cam Used in Printing and Package Machine and Linkage Mechanism (The 6th National Annual Academic Conference of Cam Mechanism) [C]. Professional Committee of Mechanism, Mechanical Transmission Institution of Chinese Mechanical Engineering Society, 2005:3.

[4]Wang Hao, Yang Yuhu, Zhang Ce. Dynamic Model and Analysis of Globoidal Indexing Cam Mechanism [J]. Mechanical Science and Technology for Aerospace Engineering , 1999,06:864-866+870.

[5]Han Xingchang. Dynamic Model and Analysis of Ball Bearing Globoidal Indexing Cam System [D].Jinan, Shandong Province: Shandong University, 2010.5: 93.

[6]He Wei, Cao Jujiang, Zhang Jianfeng, and Peng Guoxun. Dynamic Modeling and Analysis of Indexing Cam Transmission System with Rolling Teeth [J].Journal of Northwest Institute of Light Industry, 1997, 01:4-11.

[7]Cai Zhengmin, Liu Hua, Wu Haojiang. Dynamic Analysis of Globoidal Indexing Cam Mechanism [J]. Journal of Mechanical Transmission, 1999,01:4-7+53. 